\title{
Performance Analysis of SPECK Codec by DWT and IWT for Medical Image Compression
}

\author{
Revathi $\mathbf{M}^{1}$, Dr. R. Shenbagavalli ${ }^{2}$ \\ ${ }^{1}$ Research Scholar, PG \& Research Department of Computer Science, Rani Anna Government College for \\ Women, Tirunelveli-08, Affiliated to Manonmaniam Sundaranar University, India, revathi.m92@ gmail.com \\ ${ }^{2}$ Assistant Professor, PG \& Research Department of Computer Science, Rani Anna Government College for \\ Women, Tirunelveli-08, Affiliated to Manonmaniam Sundaranar University, India
}

\begin{abstract}
Image compression in medical image processing is a most significant technique which reduces the burden of storage and transmission time over the network. These medical images available in hospitals and medical organizations occupied a huge amount of space for storing these images. The compression is necessary to reduce the storage space of these images and making fast data transfer. They are classified into lossy compression and lossless compression. In this work, Magnetic Resonance Imaging (MRI) of brain and Computer Tomography (CT) of lung images are used for analyzing compression. The images are compressed using Set Partitioning Embedded block (SPECK) algorithm has been applied on Discrete Wavelet Transform (DWT) and Integer Wavelet Transform (IWT) with three wavelets such as Haar, Sym 4 and Coif1. DWT is used for lossy compression and IWT is used for lossless compression of images. The performance metrics such as Peak signal-to-noise ratio (PSNR), Structural Similarity Index (SSIM), Mean square error (MSE), Bit Per Pixel (BPP), Compression Ratio (CR) and Compression Time (CT) are measured for lung and brain images. The dataset has been collected from various scan centers.
\end{abstract}

Key words : Compression, SPECK, DWT, IWT, BPP, PSNR, SSIM, MSE,CR,CT

\section{INTRODUCTION}

Digital images are used in life applications such as television, satellite, CT, X-Ray and MRI. These images have high resolution, large size and also contain redundancy and irrelevancy information. For transmission of these images it requires large bandwidth and transmission time. Digital image compression is a method of image data reduction to save storage space. Images must be compressed before transmitting the image.
The main challenges faced by medical industry are to store images, maintain the databases of patient images and transmitting the image through the network. For this purpose the compression of images is necessary. Compression is categorized into lossy and lossless compression. In this work, the compressions of two medical image modalities such as CT and MRI are analyzed. SPECK is a wavelet based image compression technique. In this work three wavelets such as Haar, Sym 4 and Coif1 are applied in DWT-SPECK and IWT-SPECK for compression of images. DWT is a mathematical tool for decomposing an image. It does not change the information content present in the signal. IWT is used for lossless compression. Coefficients of this transform are represented by finite precision numbers and this allows for lossless coding.

\section{RELATED WORK}

Peak Signal to Noise Ratio \&Mean Square Error calculation for various Images using the lossless Image compression in CCSDS algorithm [1]. A Comparative Study of DWT, DWT-SVD and IWT-SVD", the comparative study is based on the values of the PSNR \& NCC and simulation results have been given in tabular forms [2]. It has been observed that IWT-SVD gives comparable results and faster than the DWT \& DWT-SVD. Integer wavelet transform based approach for high robustness of audio signal transmission [3] steganography is an efficient method introduced for the purpose of data hiding. An audio speech signal is transmitted at the transmitter side. ROI based medical image epitomize using SPECK and AAC this paper [4] proposes the ROI based medical image compression using SPECK and AAC for telemedicine applications. Performance Analysis of Image Compression Technique this paper addresses the area of image compression as it is applicable to various fields of image processing [5]. A Hybrid Technique for Concealing the Patient Data In Medical Images, this paper demonstrates [6] that the proposed work proved least mean square error for various biomedical images including CT scan, MRI and 
$\mathrm{X}$-rays. The proposed work framework is highly used for healthcare services [7]. Hyper spectral image compression using 3d SPIHT, speck and BEZW algorithms [8] in this paper, an analysis of efficient compression techniques is done, with more emphasis on 3D set partitioning embedded block (SPECK), binary embedded zero tree wavelet (BEZW). The dataset that is the scenes derived from standard AVIRIS hyper spectral images, which possess 224 spectral bands. The performance of the SPECK is compared with SPIHT\& JPEG2000 [9]. The quality of compression and reconstruction is measured by quantitative measures PSNR [10]. A novel morpho codec for medical video compression based on lifting wavelet transform [11] medical image compression based on set partitioning in hierarchical trees using quantized coefficients of self organizing feature map for MR images [12]. Lossless medical image compression by integer wavelet and predictive coding [13] is proposed. This would reduce the memory requirement of the embedded coding algorithm and improve the coding time to compress the images. Novel Image Compression Using Multiwavelets with SPECK Algorithm is discussed in [14]. A variation on SPECK using vector quantization to code the significant coefficients is presented in [15]. Trends in Medical Image Compression are discussed in [16]. Embedded hybrid coding for lossy to lossless image compression using integer wavelet transform is discussed in [17]. A new coding algorithm called embedded multiple subbands scaling coding (EMSSC) is presented based all reversible IWT [18]. Efficient image compression and transmission using SPECK and optimization and implementation of the integer wavelet transform for image coding [19-20].

\section{METHODOLOGY}

The input image is first transformed using DWT and IWT into multiple decomposition levels which produces different sub bands. Then, the sub bands are encoded using SPECK algorithm. The encoded bit stream is transmitted and at the receiver, it is decoded using inverse SPECK algorithm. After inverse SPECK, inverse DWT and IWT is performed on the obtained 2D sub bands. The decompressed image is used in the computation of PSNR, MSE, SSIM, CR and CT in comparison with the original image. Figure 1 shows the SPECK using DWT and IWT compression methodology.

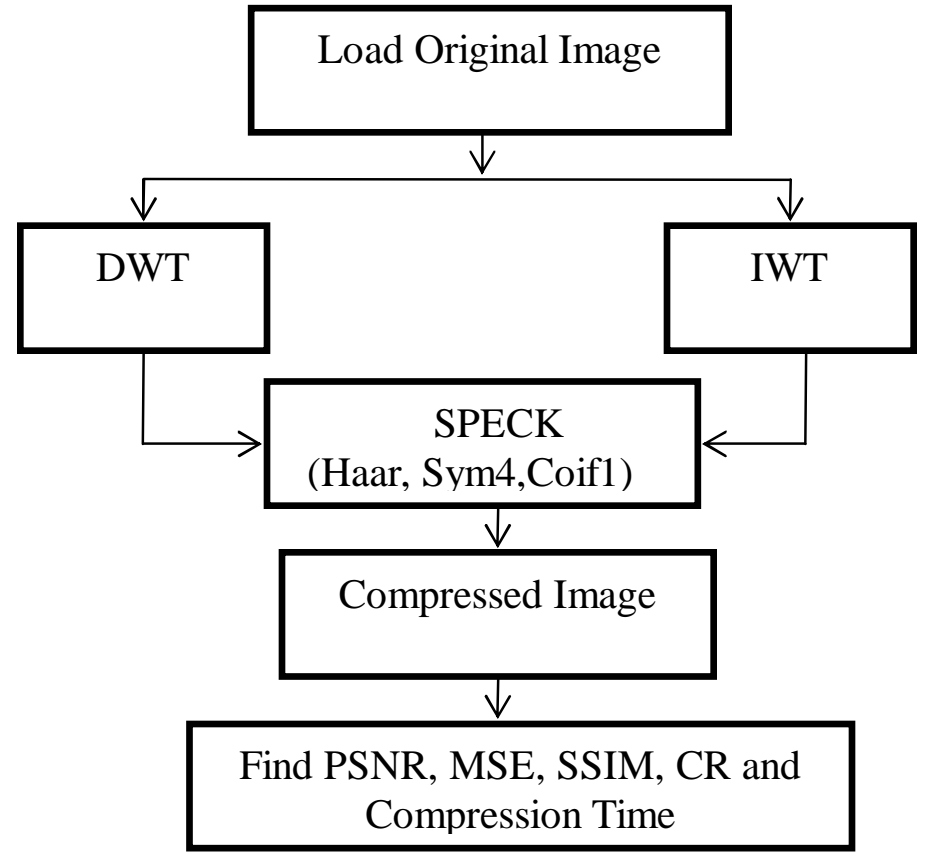

Figure 1: SPECK using DWT and IWT compression methodology

\subsection{DWT (Discrete Wavelet Transform)}

Wavelet transform decomposes a signal into a set of basis functions [20-24]. These basis functions are called wavelets. Wavelets are obtained from a single prototype wavelet called mother wavelet by dilations and shifting.

\subsection{IWT (Integer Wavelet Transform)}

The reason for choosing IWT over DWT is that IWT maps the integer data set to another integer dataset whereas DWT converts the floating point values to integer by truncation process. Truncation results in the loss of data. When the input data is in the form of an integer, perfect reconstruction is possible while applying an inverse transform. IWT is used for lossless compression. Finite precision numbers are used to represent the transform coefficients, and this allows for truly lossless coding. Image can be reconstructed without any loss because all the coefficients are integers and can be stored without rounding off errors.

\subsection{SPECK}

Set Partitioning Embedded Block Coder (SPECK).The subsets are tested for significance is important. The significance information is stored in the ordered list called (a) List of insignificant sets (LIS), (b) List of significant pixels (LSP). Each pass of SPECK comprises of sorting, and refinement. In SPECK algorithm we are using two lists than SPIHT which is a considerable improvement. A SPECK coder has a comparable performance to SPIHT but with faster implementation. 
Revathi M et al., International Journal of Advanced Trends in Computer Science and Engineering, 9(2), March - April 2020, 2451 - 2456

\section{RESULTS AND DISCUSSION}

In this section, the results of DWT-SPECK and IWT-SPECK for medical image compression are discussed. Table 1 to Table4 shows the lossy performance of IWT compared with the DWT for a set of lung and brain images. Table 1 shows the PSNR value of DWT and IWT for CT lung images and MRI brain images using three wavelets such as Haar, Sym4 and Coif1.Table 2 shows the MSE value of DWT and IWT for CT lung images and MRI brain images using three wavelets such as Haar, Sym4 and Coif1.Table 3 shows the SSIM value of DWT and IWT for CT lung images and MRI brain images using three wavelets such as Haar, Sym4 and Coif1.Table 4 shows the CT (Compression Time) value of DWT and IWT for CT lung images and MRI brain images using three wavelets such as Haar, Sym4 and Coif1. The lossless compression results of IWT are obtained in terms of achieved bit rate. Table 5 shows the lossless compression PSNR value of IWT for lung and brain images using Haar, sym4 and COIF1wavelets. Fig 2 shows the two different original lung and brain images and reconstructed images in four different BPP values. From this analysis the lung image 2 and brain image1 gives high PSNR value at 2 BPP using sym4 wavelet.

Table 1: PSNR value of DWT and IWT for brain and lung images

\begin{tabular}{|c|c|c|c|c|c|c|c|c|}
\hline \multirow[t]{3}{*}{ Images } & \multirow{3}{*}{$\mathrm{CR}$} & \multirow{3}{*}{ BPP } & \multicolumn{2}{|c|}{ Haar } & \multicolumn{2}{|c|}{ Sym 4} & \multicolumn{2}{|c|}{ COIF1 } \\
\hline & & & \multicolumn{6}{|c|}{ PSNR } \\
\hline & & & DWT & IWT & DWT & IWT & DWT & IWT \\
\hline \multirow{4}{*}{ Brain Image1 } & $32: 1$ & 0.25 & 37.66 & 32.40 & 43.13 & 36.48 & 41.23 & 31.30 \\
\hline & $16: 1$ & 0.50 & 41.42 & 35.36 & 48.67 & 38.79 & 45.99 & 32.39 \\
\hline & $8: 1$ & 1 & 46.73 & 43.28 & 54.12 & 42.43 & 51.98 & 33.08 \\
\hline & $4: 1$ & 2 & 53.75 & 48.87 & 57.69 & 56.81 & 56.97 & 33.31 \\
\hline \multirow{4}{*}{$\begin{array}{c}\text { Brain } \\
\text { Image2 }\end{array}$} & $32: 1$ & 0.25 & 36.33 & 34.23 & 42.21 & 36.26 & 39.97 & 29.68 \\
\hline & $16: 1$ & 0.50 & 39.67 & 35.75 & 47.77 & 36.52 & 44.44 & 29.70 \\
\hline & $8: 1$ & 1 & 44.58 & 42.52 & 52.70 & 40.30 & 49.84 & 31.64 \\
\hline & $4: 1$ & 2 & 50.11 & 44.90 & 55.89 & 46.82 & 54.59 & 31.69 \\
\hline \multirow[t]{4}{*}{ Lung Image1 } & $32: 1$ & 0.25 & 36.09 & 31.20 & 38.38 & 32.25 & 37.72 & 29.32 \\
\hline & $16: 1$ & 0.50 & 38.53 & 32.87 & 42.28 & 32.58 & 41.56 & 30.57 \\
\hline & $8: 1$ & 1 & 44.21 & 37.71 & 46.44 & 35.85 & 46.05 & 30.59 \\
\hline & $4: 1$ & 2 & 49.38 & 46.97 & 50.09 & 49.43 & 49.98 & 31.90 \\
\hline \multirow[t]{4}{*}{ Lung Image2 } & $32: 1$ & 0.25 & 36.29 & 32.72 & 41.08 & 33.48 & 43.64 & 30.77 \\
\hline & $16: 1$ & 0.50 & 39.47 & 35.87 & 46.50 & 35.53 & 39.39 & 31.56 \\
\hline & $8: 1$ & 1 & 44.53 & 43.18 & 51.64 & 39.60 & 48.74 & 33.69 \\
\hline & $4: 1$ & 2 & 50.20 & 47.80 & 55.75 & 44.89 & 53.47 & 33.86 \\
\hline
\end{tabular}

Table 2: MSE value of DWT and IWT for brain \& lung images

\begin{tabular}{|c|c|c|c|c|c|c|c|c|}
\hline \multirow{3}{*}{ Images } & \multirow{3}{*}{ CR } & \multirow{3}{*}{ BPP } & \multirow{2}{*}{\multicolumn{2}{|c|}{ Haar }} & \multicolumn{2}{|l|}{ Sym 4} & \multicolumn{2}{|c|}{ COIF1 } \\
\hline & & & & & \multicolumn{4}{|c|}{ MSE } \\
\hline & & & DWT & IWT & DWT & IWT & DWT & IWT \\
\hline \multirow{4}{*}{ Brain Image1 } & $32: 1$ & 0.25 & 3.34 & 6.12 & 1.78 & 3.82 & 2.21 & 6.94 \\
\hline & $16: 1$ & 0.50 & 2.16 & 4.35 & 0.94 & 2.93 & 1.28 & 6.90 \\
\hline & $8: 1$ & 1 & 1.17 & 1.75 & 0.50 & 1.93 & 0.64 & 5.66 \\
\hline & $4: 1$ & 2 & 0.52 & 0.92 & 0.33 & 0.37 & 0.36 & 5.51 \\
\hline \multirow[t]{4}{*}{ Brain Image2 } & $32: 1$ & 0.25 & 3.89 & 4.96 & 1.98 & 3.92 & 2.56 & 8.37 \\
\hline & $16: 1$ & 0.50 & 2.65 & 4.16 & 1.04 & 3.80 & 1.53 & 8.35 \\
\hline & $8: 1$ & 1 & 1.51 & 1.91 & 0.59 & 2.46 & 0.82 & 6.67 \\
\hline & $4: 1$ & 2 & 0.80 & 1.45 & 0.41 & 1.16 & 0.48 & 6.64 \\
\hline \multirow[t]{4}{*}{ Lung Image1 } & $32: 1$ & 0.25 & 4.00 & 7.02 & 3.07 & 6.22 & 3.31 & 8.72 \\
\hline & $16: 1$ & 0.50 & 3.02 & 5.79 & 1.96 & 5.99 & 2.13 & 7.54 \\
\hline & $8: 1$ & 1 & 1.57 & 3.32 & 1.21 & 4.11 & 1.27 & 7.55 \\
\hline & $4: 1$ & 2 & 0.87 & 1.14 & 0.80 & 2.72 & 0.81 & 6.48 \\
\hline \multirow[t]{4}{*}{ Lung Image 2} & $32: 1$ & 0.25 & 3.91 & 5.89 & 2.25 & 5.40 & 1.68 & 7.38 \\
\hline & $16: 1$ & 0.50 & 2.71 & 4.10 & 1.21 & 4.29 & 2.73 & 7.40 \\
\hline & $8: 1$ & 1 & 1.51 & 1.77 & 0.67 & 2.67 & 0.93 & 7.07 \\
\hline & $4: 1$ & 2 & 0.79 & 1.04 & 0.42 & 1.45 & 0.54 & 5.07 \\
\hline
\end{tabular}


Revathi M et al., International Journal of Advanced Trends in Computer Science and Engineering, 9(2), March - April 2020, 2451 - 2456

Table 3: SSIM value of DWT and IWT for brain \& lung images

\begin{tabular}{|c|c|c|c|c|c|c|c|c|}
\hline \multirow{3}{*}{ Images } & \multirow{3}{*}{$\mathrm{CR}$} & \multirow{3}{*}{ BPP } & Haar & & Sym 4 & & COIF1 & \\
\hline & & & \multicolumn{6}{|c|}{ SSIM } \\
\hline & & & DWT & IWT & DWT & IWT & DWT & IWT \\
\hline \multirow{4}{*}{ Brain Image1 } & $32: 1$ & 0.25 & 0.9085 & 0.7264 & 0.9597 & 0.7950 & 0.9516 & 0.4539 \\
\hline & $16: 1$ & 0.50 & 0.9496 & 0.8513 & 0.9858 & 0.8265 & 0.9815 & 0.4570 \\
\hline & $8: 1$ & 1 & 0.9820 & 0.9263 & 0.9960 & 0.8637 & 0.9933 & 0.5455 \\
\hline & $4: 1$ & 2 & 0.9954 & 0.9647 & 0.9979 & 0.9973 & 0.9977 & 0.5654 \\
\hline \multirow{4}{*}{ Brain Image2 } & $32: 1$ & 0.25 & 0.8771 & 0.6911 & 0.9566 & 0.7955 & 0.9389 & 0.4166 \\
\hline & $16: 1$ & 0.50 & 0.9294 & 0.8020 & 0.9841 & 0.8047 & 0.9759 & 0.4117 \\
\hline & $8: 1$ & 1 & 0.9694 & 0.9154 & 0.9947 & 0.8590 & 0.9904 & 0.4531 \\
\hline & $4: 1$ & 2 & 0.9895 & 0.9601 & 0.9971 & 0.9545 & 0.9962 & 0.5382 \\
\hline \multirow[t]{4}{*}{ Lung Image1 } & $32: 1$ & 0.25 & 0.9015 & 0.6421 & 0.9257 & 0.6360 & 0.9245 & 0.3246 \\
\hline & $16: 1$ & 0.50 & 0.9471 & 0.7384 & 0.9638 & 0.6630 & 0.9603 & 0.4047 \\
\hline & $8: 1$ & 1 & 0.9727 & 0.8869 & 0.9807 & 0.7794 & 0.9787 & 0.4157 \\
\hline & $4: 1$ & 2 & 0.9853 & 0.9685 & 0.9881 & 0.8650 & 0.9877 & 0.4793 \\
\hline \multirow[t]{4}{*}{ Lung Image2 } & $32: 1$ & 0.25 & 0.8940 & 0.7895 & 0.9541 & 0.7871 & 0.9726 & 0.4613 \\
\hline & $16: 1$ & 0.50 & 0.9379 & 0.8806 & 0.9829 & 0.8486 & 0.9384 & 0.4714 \\
\hline & $8: 1$ & 1 & 0.9708 & 0.9440 & 0.9933 & 0.9128 & 0.9892 & 0.4805 \\
\hline & $4: 1$ & 2 & 0.9899 & 0.9779 & 0.9972 & 0.9659 & 0.9953 & 0.5630 \\
\hline
\end{tabular}

Table 4: CT value of DWT and IWT for brain \& lung images

\begin{tabular}{|c|c|c|c|c|c|c|c|c|}
\hline \multirow[t]{3}{*}{ Images } & \multirow{3}{*}{$\mathrm{CR}$} & \multirow{3}{*}{ BPP } & \multicolumn{3}{|r|}{ Sym 4} & & \multicolumn{2}{|c|}{ COIF1 } \\
\hline & & & \multicolumn{6}{|c|}{ CT } \\
\hline & & & DWT & IWT & DWT & IWT & DWT & IWT \\
\hline \multirow{4}{*}{ Brain Image1 } & $32: 1$ & 0.25 & 1.86 & 1.46 & 1.67 & 1.16 & 1.11 & 1.64 \\
\hline & $16: 1$ & 0.50 & 2.50 & 3.90 & 1.87 & 2.69 & 2.89 & 4.17 \\
\hline & $8: 1$ & 1 & 1.43 & 1.85 & 1.02 & 1.48 & 1.14 & 1.52 \\
\hline & $4: 1$ & 2 & 1.53 & 1.57 & 4.52 & 5.40 & 4.39 & 4.28 \\
\hline \multirow[t]{4}{*}{ Brain Image2 } & $32: 1$ & 0.25 & 1.65 & 1.90 & 1.15 & 1.22 & 1.69 & 3.82 \\
\hline & $16: 1$ & 0.50 & 1.42 & 3.71 & 2.80 & 4.04 & 2.83 & 1.65 \\
\hline & $8: 1$ & 1 & 1.17 & 1.52 & 4.34 & 1.56 & 1.22 & 1.47 \\
\hline & $4: 1$ & 2 & 1.50 & \begin{tabular}{|l|}
1.06 \\
\end{tabular} & 5.28 & 5.82 & 4.35 & 1.30 \\
\hline \multirow[t]{4}{*}{ Lung Image1 } & $32: 1$ & 0.25 & 1.27 & 1.21 & 1.07 & 1.03 & 1.15 & 1.24 \\
\hline & $16: 1$ & 0.50 & 1.88 & 2.35 & 1.75 & 2.08 & 2.08 & 1.96 \\
\hline & $8: 1$ & 1 & 3.76 & 1.08 & 1.96 & 2.72 & 2.33 & 1.48 \\
\hline & $4: 1$ & 2 & 3.91 & 4.27 & 1.47 & 2.29 & 2.56 & 1.67 \\
\hline \multirow[t]{4}{*}{ Lung Image 2} & $32: 1$ & 0.25 & 1.42 & 1.32 & 1.18 & 1.17 & 2.15 & 1.35 \\
\hline & $16: 1$ & 0.50 & 2.63 & 2.70 & 2.38 & 2.69 & 1.16 & 3.45 \\
\hline & $8: 1$ & 1 & 1.22 & 1.20 & 2.29 & 1.23 & 1.13 & 1.44 \\
\hline & $4: 1$ & 2 & 4.33 & 5.33 & 3.29 & 3.29 & 1.46 & 1.58 \\
\hline
\end{tabular}

Table 5: Lossless performance of IWT for lung and brain images using Haar, sym4 and COIF1 wavelet

\begin{tabular}{|l|l|l|l|}
\hline \multirow{2}{*}{ Images } & \multicolumn{2}{|l|}{ Haar } & \multicolumn{2}{l|}{ Sym4 } & COIF1 \\
\cline { 2 - 4 } & \multicolumn{2}{|c|}{ Lossless Compression } \\
\hline Lung Image1 & 4.55 & 4.986 & 5.525 \\
\hline Lung Image2 & 6.55 & 5.986 & 6.55 \\
\hline Lung Image3 & 5.575 & 5.881 & 6.515 \\
\hline Lung Image4 & 4.915 & 5.916 & 6.986 \\
\hline Brain Image1 & 5.765 & 5.515 & 6.55 \\
\hline Brain Image2 & 6.55 & 5.983 & 5.575 \\
\hline Brain Image3 & 4.881 & 4.786 & 6.615 \\
\hline Brain Image4 & 5.525 & 5.578 & 6.682 \\
\hline
\end{tabular}




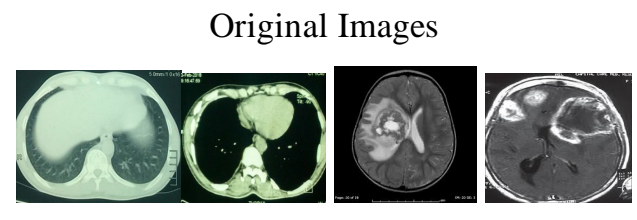

Reconstructed Images
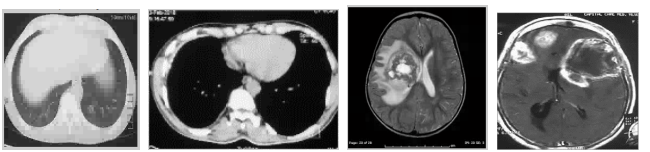

$\begin{array}{ll}\text { (i) } 0.25 & \text { (ii) } 0.50\end{array}$

(iii) 1.00

(iv) 2.00

Figure 1: SPECK using DWT and IWT compression methodology

\section{CONCLUSION}

In medical image processing, image compression is very significant because of there is huge amount of memory space is occupied in medical image. The percentage of loss of data provides improper diagnosing the patient. In this work, the lossy and lossless compression performance of the SPECK technique is analyzed with DWT and IWT. Also, three wavelets namely as Haar, Sym4, and Coif1 are analyzed on gray scale CT lung images and MRI brain images. This SPECK algorithm will results in reduced MSE, increased PSNR for lung and brain images so it can be used for transmission and storage of images. The higher PSNR value becomes better quality of the compressed or reconstructed image. The reason for choosing three wavelets such as Haar, sym 4 and Coif1among more types of wavelet families. These three wavelets only gives better PSNR for lung and brain images. Results are obtained for both lossless and lossy compression showing that the obtained IWT implementations achieve lossless compression at specific bit rate and DWT implementation gives high PSNR, less MSE and less execution time for compression of grayscale images. Experimental results are obtained for PSNR, MSE, SSIM, CR and CT. The results are compared for both CT lung images and MRI brain images. In the future work apply the SPECK coding techniques in the color image compression and in the bio-medical images.

\section{REFERENCES}

1. Ms. M. Manju, P. Abarna, U. Akila and S. Yamini, Peak Signal to Noise Ratio \&Mean Square Error calculation for various Images using the lossless Image compression in CCSDS algorithm, International Journal of Pure and Applied Mathematics, Vol. 119, pp. 14471-14477, 2018.

2. Prerna Gupta and Girish Parmar, A Comparative Study of DWT, DWT-SVD and IWT-SVD, International Journal of Electronics, Electrical and Computational System IJEECS, Vol. 6, pp. 410-415, July 2017.

3. R. Punidha, M. Sivaram, Integer Wavelet Transform Based Approach for High Robustness Of Audio Signal Transmission, International Journal of Pure and Applied Mathematics, Vol. 116, pp. 295-304, 2017.

4. P. John Vivek, K. Elangovan, R. Jayaram and B. Mohammed Javeeth, "ROI Based Medical Image
Epitomize Using SPECK and AAC, International Journal of Computational Research and Development, Vol. 1, pp. 26-29, 2016.

5. Anju, Amit Ahlawat, Performance Analysis of Image Compression Technique, International Journal of Recent Research Aspects, Vol. 3, pp. 107-111, June 2016.

6. Mappeti Kavitha, G. Vallathan and J. Asha, A Hybrid Technique for Concealing the Patient Data In Medical Images, International Journal of Modern Trends in Engineering and Science, Vol. 3, pp. 1-3, 2016.

7. Shalini Prasad, Prashant Ankur Jain and Satendra Singh, Lossless medical image compression by IWT, Pelagia Research Library, Advances in Applied Science Research, vol. 6, pp. 32-36, 2015.

8. D. Muthukumar, Hyperspectral Image Compression Using 3d Spiht, Speck and Bezw Algorithms, International Journal of Multidisciplinary Research and Modern Education, Vol. 1, pp. 242-247, 2015.

9. Pragya Tiwari ,Mohd Ahmed, S. G. Kerhalkar, Transmission of Images Using SPECK, International Journal of Computer Science and Network Security, Vol.14, pp. 97-101, November 2014.

10. D. Vijendra Babu and N. R. Alamelu, A Novel Morpho Codec for Medical Video Compression Based on Lifting Wavelet Transform, Asian Journal of Scientific Research, vol. 7, pp. 85-93, 2014.

https://doi.org/10.3923/ajsr.2014.85.93

11. S. Sridevi, V. R. vijayakumar and V. suthajebakumari, Medical Image Compression Based On Set Partitioning In Hierarchical Trees Using Quantized Coefficients Of Self Organizing Feature Map For Mr Images, Journal of Theoretical and Applied Information Technology, Vol.56, pp. 46-51, October 2013.

12. T. G. Shirsat and V. K. Bairagi, Lossless Medical Image Compression by Integer Wavelet and Predictive Coding, Hindawi Publishing Corporation ISRN Biomedical Engineering, pp. 1-6, 2013. https://doi.org/10.1155/2013/832527

13. Ruzelita Ngadiran, Said Boussakta, Ahmed Bouridane and Bayan Syarif, Hyperspectral Image Compression with Modified 3D SPECK, IEEE, pp. 806-810, 2010.

14. Sudhakar Radhakrishnan and Jayaraman Subramaniam, Novel Image Compression Using Multiwavelets with SPECK Algorithm, The International Arab Journal of 
Information Technology, Vol. 5, pp. 45-51, January 2008.

15. Chih-chien Chao and Robert M. Gray, Image Compression With A Vector Speck Algorithm, IEEE, pp. 445-448, 2006.

16. Gloria Menegaz, Trends in Medical Image Compression, Current Medical Imaging Reviews, pp. 1-21, 2006.

17. Libao Zhang, and Xianchuan Yu, Embedded Hybrid Coding for Lossy to Lossless Image Compression using Integer Wavelet Transform, IEEE TRANSACTIONS ON IMAGE PROCESSING, pp. 664-668, November 2005.

18. Zhang li-bao and Wangke, Embedded Multiple Subbands Scaling Image Coding Using Reversible Integer Wavelet Transforms, International Symposium on Intelligent Multimedia, Video and Speech Processing ,Hong Kong, pp. 599-602, October 2004.

19. W. A Pearlman, A. Islam, N. Nagaraj and A. Said, Efficient, low-complexity image coding a set partitioning embedded block coder, IEEE Transactions on Circuits and Systems for Video Technology, Vol.14, pp.1219-1239, November 2004. https://doi.org/10.1109/TCSVT.2004.835150

20. N.B. Chopade, A. A. Ghatol and M. T. Kolte, Efficient Image Compression and TransmissionUsing SPECK, Proceedings of SPIT-IEEE Colloquium and International Conference, Mumbai, India, Vol. 1, pp. 156-160, 2004.

21. R.R Mohamed, M.A. Mohamed, K. Azmi, E. Rao, W. Hashim, Indoor smart lighting controlling using human detection. International Journal of Advanced Trends in Computer Science and Engineering, vol. 9, pp. 566-570, 2020.

22. B. Kishore, V.V. Kumar, Gradient ternary transition based cross diagonal texture matrix for texture classification. International Journal of Advanced Trends in Computer Science and Engineering, vol. 8, pp. 1332-1339. https://doi.org/10.30534/ijatcse/2019/47842019

23. S. Kumarapandian, Melanoma Classification Using Multiwavelet Transform and Support Vector Machine. International Journal of MC Square Scientific Research, vol. 10, pp. 01-07, 2018.

24. S. Murugan, A. Bhardwaj, T.R. Ganeshbabu, Object recognition based on empirical wavelet transform. International Journal of MC Square Scientific Research, Vol. 7, pp. 74-80, 2015.

https://doi.org/10.20894/IJMSR.117.007.001.008 